\title{
Factors affecting survival in neonatal surgery unit in a tertiary care university hospital during 26 years
}

\author{
Önder Özden, İbrahim Karnak, Arbay Özden Çiftçi, F. Cahit Tanyel, Mehmet Emin Şenocak \\ Department of Pediatric Surgery, Hacettepe University Faculty of Medicine, Ankara, Turkey. \\ E-mail: ikarnak@hacettepe.edu.tr \\ Received: 23rd March 2016, Accepted: 23rd January 2017
}

SUMMARY: Özden Ö, Karnak İ, Çiftçi AÖ, Tanyel FC, Şenocak ME. Factors affecting survival in neonatal surgery unit in a tertiary care university hospital during 26 years. Turk J Pediatr 2016; 58: 457-463.

This clinical study was designed to evaluate mortality rate and the factors that may affect survival in neonatal surgery unit.

Randomly chosen 300 ( $($ : 0.20 ) patients among 1,439 patients treated in neonatal surgery unit during years 1983 to 2009, were evaluated retrospectively. The patients were separated into three groups according to date of treatment; Group A: 1983 - 1995, Group B: 1996 - 2005 and Group C: 2005 - 2009. M/F ratios did not differ between non-survived and survived patient populations. Mortality rates were $37 \%, 22 \%$ and $13 \%$ in Group A, B, and C respectively $(\mathrm{p}<0.001)$. Parenteral nutrition, maternal age, time until admission and gestational age did not affect mortality rate, however median age of newborn was lower in non-survived cases ( 1 day vs. 3 days, $\mathrm{p}<0.001)$. Associating abnormality, low birth weight $(<1,500 \mathrm{~g})$, associating sepsis, need of globulin and requirement of respiratory support were determinants of lower survival $(\mathrm{p}<0.001)$. The mortality rate for patients that underwent thoracotomy (42\%) and laparotomy $(41 \%)$ were higher than patients that underwent other operations $(8 \%)$ and observation $(10 \%)$ $(\mathrm{p}<0.001)$. Diaphragmatic hernia had higher mortality rates than the other pathologies $(p<0.001)$. Survival rate is increasing to date in newborn pediatric surgery unit; it is independent from parenteral nutrition, maternal age, time to admission and gestational age however it is affected adversely by the age of patient, associating abnormality, low birth weight, presence of sepsis and requirement of respiratory support. Increase in survival could be related to various additional factors such as development of delicate respiratory support machines, broad spectrum antibiotics, hospital infection control teams, central venous catheters, use of TPN by central route, volume adjustable infusion pumps, monitoring devices, neonatal surgical techniques, prenatal diagnosis of pediatric surgical conditions and developments of environmental control methods in neonatal surgical units.

Key words: newborn, pediatric surgery, mortality, survival.

Newborn period is the interval time from birth to 28-day. William Ladd established newborn surgery, 81 years ago. Newborn mortality rate in 1940's and 1950's was very high in Boston Children Hospital, in spite of being one of the best centers of pediatric surgery. Many developments and innovations caused a remarkable reduction of newborn mortality rate. The newborn mortality rate was dropped from $100 \%$ to $10 \%$ during this circumstance ${ }^{1}$. The aims of this study were the determination of mortality rates and the parameters affecting survival in neonatal surgery unit.

\section{Material and Methods}

Infants treated in Newborn Surgery Unit of Pediatric Surgery Department of Hacettepe University hospital from 1983 to 2009 inclusive (n: 1439) enrolled study. The patient population were randomly sampled for 300 infants $(B$ : $0.20)$. This sample population was divided 
into 3 respective groups according to date of hospitalization: Group A, 1983 - 1995; Group B, 1996 - 2005, Group C, 2005 - 2009. Mortality rate, gender, elapsed time from the appearance of symptoms till hospital admission, age at admission, maternal age, gestational age, birth weight, surgical diagnosis, associating abnormalities, sepsis status, results of microbial cultures, gamma globulin usage, mechanical ventilation requirement and total parenteral nutrition (TPN) treatment status were noted. The data were expressed as mean \pm SD or median (range) when available and Student's $t$ test, Mann-Whitney U test and Chi-square test were used for statistical analyses. A p value lower than 0.05 was considered significant.

\section{Results}

One thousand four hundred thirty-nine newborns were admitted to Newborn Surgery Unit of Pediatric Surgery Department between 1983 and 2009. Total mortality rate was $30 \%$ (n: 441) in this period. The mortality rates for Group A, Group B and Group C were 37\% (337/907), 22\% (80/352) and 13\% (24/180), respectively $(\mathrm{p}<0.001$, Fig. 1$)$.

Male to female ratio was 1.67 in the sample patient population. Male to female ratios did not differ between non-survived and survived patient populations (1.64 vs. 1.69 , p: 0.916). $\mathrm{M} / \mathrm{F}$ ratios were $1.78,1.77$ and 1.18 for Groups $\mathrm{A}, \mathrm{B}$ and $\mathrm{C}$, respectively, in survived patients. The ratios were 1.55, 1.62 and 3 for Groups A, $\mathrm{B}$ and $\mathrm{C}$, respectively, in non-survived patients. The gender difference was not statistically significant in groups.

Median ages at admission are presented in Table
I. Maternal age was not available in 17 patients. It was $26.08 \pm 5.4$ years and $26.13 \pm 5.4$ years in the survived and non-survived patient populations without expressing significant difference ( $\mathrm{p}$ : 0.776). The newborn mortality rates were $32 \%$ and $37 \%$ for maternal age below 35 years and above 35 years, respectively. The mortality rates did not differ when 35 -year of maternal age was chosen as a cut-off level (p: 0.741). Maternal age in Groups A, B and $\mathrm{C}$ was $24.92 \pm 4.9$ years, $27.74 \pm 6.2$ years and $27.00 \pm 4.8$ years in survived patients; and $25.41 \pm 5.1$ years, $27.24 \pm 5.4$ years and $28.88 \pm 7.1$ years in respective groups in non-survived patients. The maternal age did not differ in Groups A, B and C in survived and non-survived patients (p: $0.465, \mathrm{p}: 0.745$ and p: 0.334).

Birth weight information was lacking in 18 patients. The mortality rates were $75 \%$ and $31 \%$ in patients with a birth weight under $1,500 \mathrm{~g}$ and above $1,500 \mathrm{~g}$. The mortality rate was significantly higher in patients with a birth weight below 1,500 g (p: 0.015). Birth weight of patients in Groups A, B and C was $2,988 \pm 509 ; 3,053 \pm 657$ and $2,915 \pm 787 \mathrm{~g}$ in survived patients and 2,788 $\pm 679 ; 2,633$ \pm 797 and $2,812 \pm 343 \mathrm{~g}$ in corresponding groups of non-survived patients. The birth weights did not differ in Groups A and Groups $\mathrm{C}$ in survived and non-survived patients ( $\mathrm{p}$ : 0.073, p: 0.614). However, birth weight was significantly lower in Group B in non-survived patients (p: 0.019).

Gestational age data was not available in 29 patients. Gestational age was $38.54 \pm 2.5$ weeks and $37.93 \pm 3.1$ weeks in survived and non-survived patients without significance ( $\mathrm{p}$ :

Table I. Survival Status of Sample Population with Respect to Patient Age.

\begin{tabular}{lccccc}
\hline & & & & Age (day) & p \\
\hline \multirow{2}{*}{ Sample } & Survived & 3.00 & 4.28 & $0-24$ & \\
& Nonsurvived & 1.00 & 2.03 & $0-15$ & $<0.001$ \\
\multirow{2}{*}{ Group A } & Survived & 3.00 & 3.90 & $0-24$ & \\
& Nonsurvived & 2.00 & 2.13 & $0-10$ & $<0.001$ \\
\multirow{2}{*}{ Group B } & Survived & 1.00 & 3.92 & $0-24$ & \\
\multirow{2}{*}{ Group C } & Nonsurvived & 1.00 & 2.48 & $0-15$ & 0.446 \\
& Survived & 6.50 & 7.04 & $0-24$ & \\
& Nonsurvived & 0.00 & 0.00 & 0 & $<0.001$ \\
\hline
\end{tabular}


$0.184)$. The median value of gestational age was 40, 40 and 38 weeks and 40, 37 and 38.5 weeks in respective groups of $\mathrm{A}, \mathrm{B}$ and $\mathrm{C}$ in survived and non-survived patients. Gestational age did not differ in Groups A, B and C of survived and non-survived patients (p: 0.093, p: 0.106, p: 0.593).

Associating abnormality was encountered in $37 \%$ and $11 \%$ of patients in non-survived and survived group, respectively. The rate of associating abnormalities was significantly elevated in non-survived patients $(\mathrm{p}<0.001)$. The most frequent associating abnormality was cardiac malformation. The rates of associating abnormalities concerning to Group A, B and C were $23.2 \%, 71.4 \%$ and $75.0 \%$ in non-survived patients. They were significantly elevated when compared with Group A, B and C (7.7\%, $13.1 \%$, and $20.8 \%$ ) in survived patients ( $\mathrm{p}$ : $0.006, \mathrm{p}<0.001$ and $\mathrm{p}: 0.018)$.

Mechanical ventilation support was used in 39 $(67 \%)$ and $19(9 \%)$ patients in non-survived and survived patient groups respectively $(\mathrm{p}<0.001)$. Concerning to Group A, B and C, the rates of mechanical ventilation requirement in non-survived and survived patients were $20 \%$ vs. $3 \%, 81 \%$ vs. $15 \%$ and $100 \%$ vs. $29 \%$. The rate of mechanical ventilation support use was significantly elevated in all groups in non-survived patients $(\mathrm{p}<0.001, \mathrm{p}<0.001$, and $\mathrm{p}<0.001)$.

The mortality rates in patients with sepsis were $75 \%$ (n: 65), and $15 \%$ (n: 33) in patients without sepsis. The presence of sepsis was found to be associated with mortality $(\mathrm{p}<0.001)$. The mortality rates according to the presence of sepsis in groups were as follow; Group A ( $80 \%$ vs. 20\%, p<0.001), Group B $(86 \%$ vs. $5 \%, \mathrm{p}<0.001)$ and Group C $(56 \%$ vs. $13 \%$, p: 0.023$)$. Increased mortality rates were associated with the presence of sepsis in all groups.

Forty-two percent (n: 33) of patients with positive microbial culture result (at least one positive result for throat, blood, urine, stool, tracheal aspirate, surgical wound) and 29\% (n: 65) of patients without positive culture result did not survive. The mortality rates in patients with or without positive culture according to groups were as follow; Group A ( $46 \%$ vs. $33 \%$, p: 0.097), Group B (33\% vs. $24 \%$, p: 0.516$)$ and Group C (38\% vs. $21 \%$, p: 0.378). The presence of positive microbial culture result did not affect survival of patients either in the whole sample or in groups.

Median value of the time elapsed from the start of symptoms to admission was 24 hours in both survived and non-survived patients (p: 0.320 ). Median value of the time elapsed until admission in survived and non-survived patients in groups was as follow; Group A (48 hours vs. 48 hours, p: 0.088), Group B (12 hours vs. 12 hours, p: 0.702) and Group C (24 hours vs. 0 hour, p: 0.070). The time elapsed until admission had no effect on survival.

The rate of TPN administration was $13 \%$ (n: 13 ) and $14 \%$ (n: 29) in non-survived and survived groups, respectively. The use of TPN did not have an effect on survival (p: 0.938). The use of TPN in survived and non-survived

Table II. Diagnosis Specific Mortality Rates of Patients in Each Group.

\begin{tabular}{lccccc}
\hline Diagnosis & Total & Group A & Group B & Group C & $\mathrm{p}$ \\
\hline Esophageal atresia & $24 / 49(48 \%)$ & $19 / 34(56 \%)$ & $4 / 11(36 \%)$ & $1 / 4(25 \%)$ & 0.312 \\
Anorectal malformation & $12 / 64(18 \%)$ & $10 / 48(20 \%)$ & $2 / 14(14 \%)$ & $0 / 2(0 \%)$ & $*$ \\
Omphalocele & $14 / 37(37 \%)$ & $11 / 24(45 \%)$ & $2 / 11(18 \%)$ & $1 / 2(50 \%)$ & 0.250 \\
Aganglionic megacolon & $1 / 13(7 \%)$ & $1 / 10(10 \%)$ & $0 / 1(0 \%)$ & $0 / 1(0 \%)$ & $*$ \\
Intestinal atresia & $9 / 26(34 \%)$ & $6 / 16(37 \%)$ & $1 / 5(20 \%)$ & $2 / 5(40 \%)$ & 0.689 \\
Diaphragmatic hernia & $14 / / 20(70 \%)$ & $4 / 5(80 \%)$ & $8 / 12(66 \%)$ & $2 / 3(66 \%)$ & 0.846 \\
Necrotizing enterocolitis & $4 / 10(40 \%)$ & $3 / 6(50 \%)$ & $1 / 1(100 \%)$ & $0 / 3(0 \%)$ & $*$ \\
Intestinal obstruction & $13 / 34(38 \%)$ & $11 / 21(52 \%)$ & $1 / 10(10 \%)$ & $1 / 3(33 \%)$ & 0.069 \\
Other diagnoses & $7 / 47(14 \%)$ & $4 / 21(19 \%)$ & $2 / 17(11 \%)$ & $1 / 9(11 \%)$ & 0.772 \\
\hline
\end{tabular}

* Because of cell frequencies, expected value less than 5 in cross tables, were above $20 \%$, statistical results could not be given. 
patients in groups as follows; Group A (6\% vs. $4 \%$, p: 0.747$)$, Group B (23\% vs. $23 \%$, p: $1.00)$ and Group C (33\% vs. $23 \%$, p: 0.219). The rate of TPN administration did not differ between survived and non-survived patients.

Gamma globulin was used in $12 \%$ (n: 25) and $36 \%$ (n: 35 ) of patients in survived and non-survived patient groups $(p<0.001)$. The rate of gamma globulin use in survived and non-survived patients according to the groups were as follows; Group A ( $13 \%$ vs. $26 \%$, p: $0.037)$, Group B ( $8 \%$ vs. $57 \%, \mathrm{p}<0.001)$ and Group C (21\% vs. 63\%, p: 0.072). Gamma globulin usage was higher when taking the whole sample into consideration and in each earlier groups; Group A and Group B in nonsurvived patient population.

Thoracotomy, laparotomy, genitourinary, other operations and only observation rates were $11.7 \%$ (n: 35$), 60 \%$ (n: 180), 10\% (n: 30), 6\% (n: 18$)$ and $12.3 \%$ (n: 37 ) in the whole sample. The type of treatment was thoracotomy $(15.3 \%$, $\mathrm{n}: 15)$, laparotomy $(76.5 \%, \mathrm{n}: 75)$, other operations including genitourinary operations (4.1\%, n: 4) and observation (4.1\%, n: 4) in non-survived patients. The mortality rate was higher in patients undergone laparotomy and thoracotomy when compared with other operations and observation $(p<0.001)$. The survival rates in patients treated by thoracotomy or laparotomy did not differ (p: 0.724) (Fig. 2).

The distribution of type of treatments was laparotomy $(62.4 \%, \mathrm{n}: 116)$, thoracotomy (12.3\%, n: 23$)$, other operations $(8.1 \%, \mathrm{n}: 15)$ and observation $(8.6 \%, \mathrm{n}: 16)$ in Group A. The distribution was laparotomy $(78.3 \%, \mathrm{n}: 54)$, thoracotomy $(15.9 \%, \mathrm{n}: 11)$, other operations $(2.9 \%, \mathrm{n}: 2)$ and observation (2.9\%, n: 2) in non-survived patients. The mortality rate was higher in patients who had undergone laparotomy than other treatments in Group A $(\mathrm{p}<0.001)$.

The distribution of the type of treatment was laparotomy $(57.3 \%$, n: 47$)$, thoracotomy (11\%, n: 9), other operations (15.9\%, n: 13$)$ and observation $(15.9 \%$, n: 13$)$ in Group B. The treatment was laparotomy $(66.7 \%, \mathrm{n}: 14)$, thoracotomy $(19 \%, \mathrm{n}: 4)$, other operations (9.5\%, n: 2) and observation (4.8\%, n: 1$)$ in non-survived patients. The mortality rates did not differ between modes of treatments in Group B.
The treatment was laparotomy (53.1\%, n: 17$)$, thoracotomy $(9.4 \%, \mathrm{n}: 3)$, other operations $(12.5 \%, \mathrm{n}: 4)$ and observation $(25 \%, \mathrm{n}: 8)$ in Group C patients. Seven patients had undergone laparotomy and one patient under clinical observation died in this group. Statistical analyses could not be applied in Group C.

Mortality rates are presented in Table 2. Patients with diagnosis of diaphragmatic hernia had higher mortality rates than the other diseases $(\mathrm{p}<0.001)$ (Table II and Fig. 3). Patients with diaphragmatic hernia had higher mortality rates than the others in Group A $(\mathrm{p}<0.001)$. Statistical analysis results were not presented because cell frequencies were above $20 \%$ and the expected value was less than 5 in cross tables in Group B and C (Table II).

\section{Discussion}

There is no extensive data about newborn mortality rates in neonatal surgery units, in the English language medical literature. The newborn mortality rate was $72 \%$ in $1949^{2}$ and it can be supposed to be around $10 \%$ at the present time. It has been reported that newborn mortality rate was $9 \%$ in surgery units in Japan in $2003^{3}$. General newborn mortality rate in Turkey was $25.78 / 1000$ alive birth, that in Japan was $2.79 / 1000$ alive birth in $2009^{4}$. Although general newborn mortality rate in Turkey was 10 times higher than that in Japan, newborn mortality rate in our institution was almost the same as in Japan. This showed that our institution was in the same level with that developed country.

It has been reported that $63 \%$ of patients in newborn surgery unit was male ${ }^{5}$. In the present study, $\mathrm{M} / \mathrm{F}$ ratio was found to be 1.66 . To the best of our knowledge, gender has no impact on the mortality rate in newborn surgical units.

It has been reported previously that the mortality rates were $45 \%, 12 \%$ and $5 \%$ in newborns with a birth weight range of 501 - 750 grams, 751 - 1,000 grams and 1,001 1,500 grams respectively 6 . There is a simple adverse relation between birth weight and mortality rate. The higher the birth weight, lower mortality rates can be expected. The mortality rate was found significantly elevated in newborns with birth weight below 1,500 grams in the present study. 


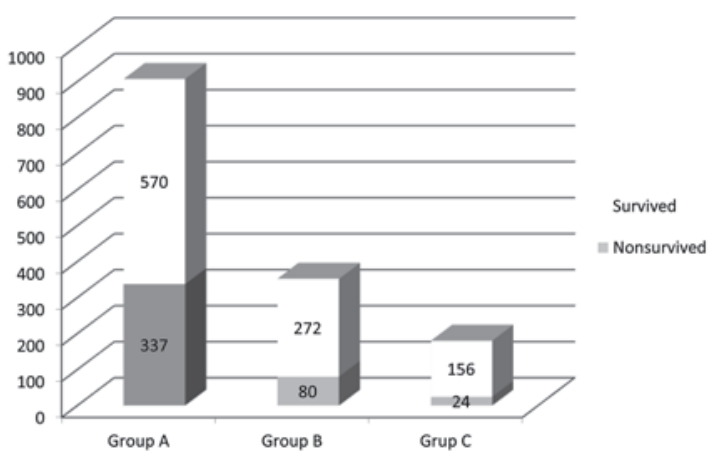

Fig. 1. Survival status of patients.

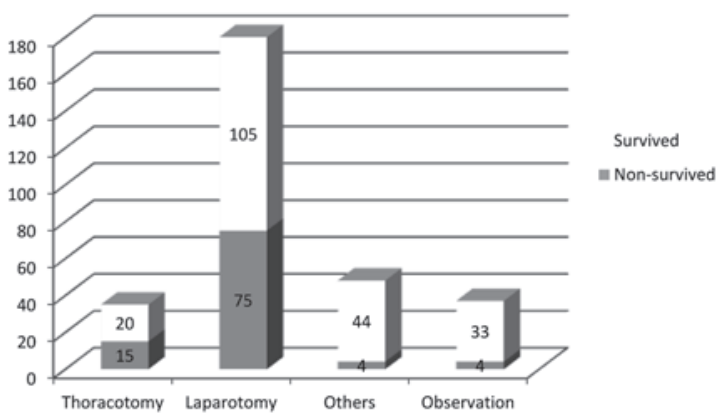

Fig. 2. Survival status of patients with respect to type of operation.

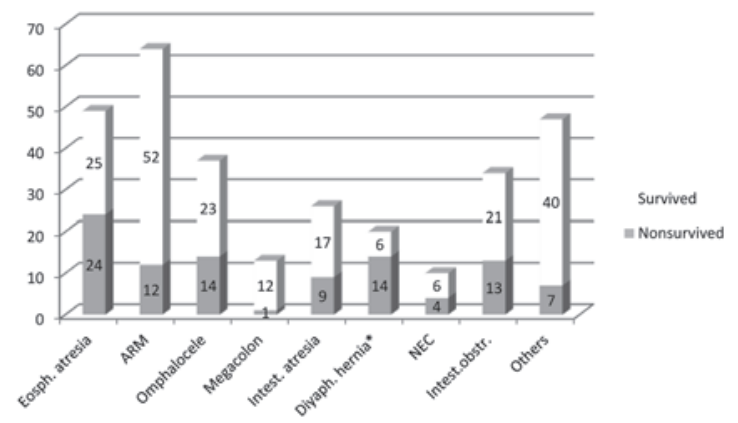

Fig. 3. Pathology specific survival of patients (ARM: Anorectal malformation; NEC: Necrotizing enterocolitis).

It has been reported that, mortality rate among newborns born to mothers younger than 20 years of age was 10.3 per 1000 alive birth when the general mortality rate at the same time interval was 7.0 per 1000 alive birth ${ }^{7}$. Additionally, infant mortality rate was found to be 2.18 times higher among children whose mothers were aged 15 years old when compared to children whose mothers were between 24-29 years of age ${ }^{8}$. Maternal age did not affect the newborn mortality rate in the present study. This may be due to higher maternal ages in our study population or possibly extensive support of grandmothers to young mothers in the closed family structure in our country.

Lower gestational age has been reported to be predisposing factor for newborn deaths. Lower gestational age has been associated with higher mortality rates in newborns ${ }^{9,10}$. This parameter could not have been investigated sufficiently in the present study because of limited numbers of mothers having significantly lower gestational aged babies.

Elapsed time between initiation of symptoms and arrival to hospital can be supposed to play role in the newborn mortality rates. However, it has been found that this parameter had no significant effect on the mortality rate in surgical newborns in the present study similar to previous report ${ }^{11}$.

The mortality rate for gastroschisis was $100 \%$ in 1953. However, it decreased to $10 \%$ in 1990 's ${ }^{2,12}$. Improvement in newborn survival rates was encountered during study period in the present study. Mortality rate decreased to $25 \%$ from $55 \%$ in surgical newborns with esophageal atresia ${ }^{13}$. Similar improvement was also observed in newborn with abdominal wall defects. The mortality rate was $45 \%$ in earlier dates. It was decreased to $18 \%$ in the next time period in our study. Various developments in respiratory support, vascular access and nutrition of surgical newborn played role in amelioration of survival rates.

Caloric intake is mandatory in humans. Newborns cannot live more than a few weeks without caloric intake ${ }^{14}$. Unfortunately, enteral feeding has to be held in many surgical newborns that undergo operations for NEC, intestinal atresia and abdominal wall defect. Development of parenteral nutrition solutions and central vascular access devices started a new era in the care of surgical newborns ${ }^{15}$. The frequency of TPN usage increased gradually during the study period in our newborn surgery clinic. The rate of TPN use was similar in survived and non-survived patient populations in our study. Therefore, we could not make a definite conclusion on the effect of TPN administration.

Respiratory support machines and ventilation techniques were also well developed over time. The initial mechanical ventilator machine was 
Baby bird 1, which was introduced in 1971 . Continuous positive airway pressure (CPAP) and intermittent mandatory ventilation (IMV) were innovated later ${ }^{16,17}$. The use of mechanical ventilation was higher in the non-survived patient population in our study, which was as expected.

Sepsis is another important parameter that affects survival in newborns ${ }^{18,19}$. The incidence of sepsis was higher in the nonsurvived population in the present study. This observation is not a surprise since sepsis is accompanied in most cases of death.

Gamma globulin has been reported to reduce early newborn mortality rates but not affect general mortality rates in newborns with gestational age less than 34 weeks and weighing between $500-2,000$ grams $^{20}$. It has been used previously to support immune systems in critically ill surgical newborns. The association between gamma globulin usage and high mortality rate in the present research is due to its selective use in critically ill patients. Nowadays, gamma globulin is not administered as frequently as used previously.

Abdominal and thoracic operations are heavier than urological operations and may necessitate specific treatment modalities such as TPN and respiratory support with mechanical ventilation. Abdominal and thoracic operations were found associated with higher mortality rates as expected, in our study. Congenital diaphragmatic hernia is a serious disease and it should be evaluated as a separate pathology. It is permanently associated with high mortality rate in this study. The mortality rate in $\mathrm{CDH}$ may decrease in the future with the use of advanced treatment methods such as extracorporeal membrane oxygenation and new ventilation techniques as well as new pharmacological agents acting on pulmonary capillary bed.

One of the limitations of this study was its retrospective design. Although power analyses were done and sample number was determined according to selected $ß: 0.20$ value, some statistical calculations could not be done. The other limitation is the method of sepsis diagnosis. It was diagnosed clinically, not through detailed criteria of sepsis.

Survival is increasing to date in newborn pediatric surgery unit. The survival is independent from parenteral nutrition, maternal age, time to admission and gestational age however it is affected adversely by the age of patient, associating abnormality, low birth weight, presence of sepsis and requirement of respiratory support.

The decrease of mortality rates in neonatal surgical units depends on many factors changing in years such as development of delicate respiratory support machines that can generate various modes of ventilations, broad spectrum antibiotics, intensive work of hospital infection control teams, delicate central venous access catheters, use of TPN, increased caloric fact of parenteral feeding solutions, use of TPN by central route, volume adjustable infusion pumps, noninvasive and invasive monitoring devices, development of neonatal surgical techniques, development in prenatal diagnosis of pediatric surgical conditions and developments of environmental control methods in neonatal surgical units.

\section{REFERENCES}

1. Hendren WH, Lillehei CW. Pediatric surgery. N Engl J Med 1988; 319: 86-96.

2. Rowe MI, Rowe SA. The last fifty years of neonatal surgical management. Am J Surg 2000; 180: 345-352.

3. Watanabe Y. Trends in neonatal surgery in Japan. Nihon Geka Gakkai Zasshi 2007; 108: 313-317.

4. The World Factbook 2009. Washington, DC: Central Intelligence Agency 2009; https://www.cia.gov/library/ publications/the-world-factbook/index.html.

5. Badawi N, Adelson P, Roberts C, Spence K, Laing S, Cass D. Neonatal surgery in New South Wales--what is performed where? J Pediatr Surg 2003; 38: 10251031.

6. Fanaroff AA, Stoll BJ, Wright LL, et al. Trends in neonatal morbidity and mortality for very low birthweight infants. Am J Obstet Gynecol 2007; 196: 147. e1-e8.

7. Chen XK, Wen SW, Fleming N, Yang Q, Walker MC Increased risks of neonatal and postneonatal mortality associated with teenage pregnancy had different explanations. J Clin Epidemiol 2008; 61: 688-694.

8. Friede A, Baldwin W, Rhodes $\mathrm{PH}$, et al. Young maternal age and infant mortality: the role of low birth weight. Public Health Rep 1987; 102: 192-199.

9. Battaglia FC, Lubchenco LO. A practical classification of newborn infants by weight and gestational age. J Pediatr 1967; 71: 159-163.

10. McConnochie KM, Roghmann KJ, Liptak GS. Avoidable morbidity in infants. A classification based on diagnoses in administrative databases. Med Care 1997; 35: 237 254. 
11. Arslankoylu AE, Bayrakci B, Oymak Y. Admission time and mortality rates. Indian J Pediatr 2008; 75 : 691-694.

12. Moore TC, Stokes GE. Gastroschisis; report of two cases treated by a modification of the gross operation for omphalocele. Surgery 1953; 33: 112-120.

13. Spitz L. Esophageal atresia: past, present, and future. J Pediatr Surg 1996; 31: 19-25.

14. Heird WC, Driscoll JM, Jr., Schullinger JN, Grebin $\mathrm{B}$, Winters RW. Intravenous alimentation in pediatric patients. J Pediatr 1972; 80: 351-372.

15. Dalla Vecchia LK, Grosfeld JL, West KW, Rescorla FJ, Scherer LR, Engum SA. Intestinal atresia and stenosis: a 25-year experience with 277 cases. Arch Surg 1998; 133: 490-496; discussion 496-497.
16. Kirby RR. Intermittent mandatory ventilation in the neonate. Crit Care Med 1977; 5: 18-22.

17. Scopesi F, Calevo MG, Rolfe P, et al. Volume targeted ventilation (volume guarantee) in the weaning phase of premature newborn infants. Pediatr Pulmonol 2007; 42: $864-870$.

18. Martius JA, Roos T, Gora B, et al. Risk factors associated with early-onset sepsis in premature infants. Eur J Obstet Gynecol Reprod Biol 1999; 85: 151-158.

19. Darmstadt GL, Bhutta ZA, Cousens S, Adam T, Walker N, de Bernis L. Evidence-based, cost-effective interventions: how many newborn babies can we save? Lancet 2005; 365: 977-988.

20. Weisman LE, Stoll BJ, Kueser TJ, et al. Intravenous immune globulin prophylaxis of late-onset sepsis in premature neonates. J Pediatr 1994; 125(6 Pt 1): 922930. 